\title{
Prolonged Clinical Benefit of Everolimus Therapy in the Management of High-Grade Pancreatic Neuroendocrine Carcinoma
}

\author{
Paula J. Fonseca ${ }^{a}$ Esther Uriol ${ }^{a}$ José A. Galván ${ }^{b}$ Carlos Álvarez $^{a}$ \\ Quionia Pérez $^{a} \quad$ Noemi Villanueva ${ }^{a}$ José P. Berros ${ }^{a}$ Marta Izquierdo ${ }^{a}$ \\ José M. Viéitez ${ }^{\mathrm{a}}$ \\ ${ }^{a}$ Medical Oncology Department, Hospital Universitario Central de Asturias, and \\ ${ }^{b}$ Department of Pathology, University Institute of Oncology, University of Oviedo, \\ Oviedo, Spain
}

\section{Key Words}

Neuroendocrine tumors · Pancreatic · Everolimus · Octreotide long-acting repeatable

\begin{abstract}
Treatment options for patients with high-grade pancreatic neuroendocrine tumors (pNET) are limited, especially for those with progressive disease and for those who experience treatment failure. Everolimus, an oral inhibitor of mammalian target of rapamycin (mTOR), has been approved for the treatment of patients with low- or intermediate-grade advanced pNET. In the randomized phase III RADIANT-3 study in patients with low- or intermediategrade advanced pNET, everolimus significantly increased progression-free survival (PFS) and decreased the relative risk for disease progression by $65 \%$ over placebo. This case report describes a heavily pretreated patient with high-grade PNET and liver and peritoneal metastases who achieved prolonged PFS, clinically relevant partial radiologic tumor response, and resolution of constitutional symptoms with improvement in Karnofsky performance status while receiving a combination of everolimus and octreotide long-acting repeatable (LAR). Radiologic and clinical responses were maintained for 19 months, with minimal toxicity over the course of treatment. This case supports the findings that the combination of everolimus plus octreotide LAR may be considered for use in patients with high-grade PNET and progressive disease. Although behavior and aggressiveness are different between low-
\end{abstract}


Fonseca et al:: Prolonged Clinical Benefit of Everolimus Therapy in the Management of High-Grade Pancreatic Neuroendocrine Carcinoma

or intermediate-grade and high-grade pNET, some high-grade pNET may express mTOR; hence, everolimus should be considered in a clinical trial.

(C) 2013 S. Karger AG, Basel

\section{Introduction}

Pancreatic neuroendocrine tumors (pNET), neoplasms that arise from cells of the endocrine system, represent approximately $1 \%$ of all cases of pancreatic cancer by incidence and $9 \%$ by prevalence in the United States [1]. In the European Union, the incidence is estimated to be $5.25 / 100,000$ [2]. Prognosis is generally poor because the diagnosis is frequently unresectable or metastatic disease. Patients with low- or intermediate-grade advanced disease have a median survival time of 24 months; for those with poorly differentiated (highgrade) pNET, median survival time is even shorter ( $\leq 10$ months) [1,3]. Current clinical aims focus on prolonging progression-free survival (PFS) for patients with advanced pNET while minimizing treatment-related toxicities.

Classic European guidelines for the management of advanced low-grade pNET recommend streptozocin plus 5-fluorouracil/doxorubicin or, currently, temozolomide, with or without capecitabine, as chemotherapy, and everolimus or sunitinib as targeted agents. Although treatment with chemotherapy results in response rates of $\sim 35 \%$, it is associated with severe toxicity $[2,4-7]$. Once patients experience treatment failure, therapeutic options are limited.

As in patients with low-grade NET, recommendations for first-line therapy in patients with high-grade tumors include chemotherapy that is based on the pattern of small cell lung cancer with etoposide and platinum [8]. Although patients with high-grade advanced tumors have the highest response rates, the duration of response is often short, and there is little evidence of benefit beyond 4 treatment cycles [8]. Retreatment with chemotherapy after relapse occurring 3-6 months following the first treatment may be considered but has not been investigated in trials [8].

Everolimus, an orally administered mammalian target of rapamycin (mTOR) inhibitor, has demonstrated both clinical efficacy and acceptable toxicity in patients with low- or intermediate-grade advanced pNET [9]. Positive results from 2 phase II studies subsequently prompted further investigation in this patient group with the prospective, randomized, phase III RADIANT-3 trial [10]. RADIANT-3 was conducted to assess the efficacy and safety of everolimus $(10 \mathrm{mg})$ compared with placebo among patients with low- or intermediategrade advanced pNET and radiologic documentation of disease progression [10]. Results showed median PFS to be significantly longer among patients receiving everolimus than among those receiving placebo [11.0 vs. 4.6 months; hazard ratio, $0.35 ; 95 \%$ confidence interval (CI), 0.27-0.45; $\mathrm{p}<0.001$ ). The objective response rate by Response Evaluation Criteria In Solid Tumors version 1.0 (RECIST v1.0) was also higher with everolimus than with placebo (all partial responses: 5 vs. $2 \%$, respectively). Stable disease was observed in $73 \%$ of everolimus-treated patients compared with $51 \%$ of those receiving placebo, and tumor shrinkage was also greater in the everolimus arm (64\%) compared with the placebo arm (21\%). Everolimus was generally well tolerated; adverse events were primarily grade $1 / 2$ in severity, consistent with known mTOR side effects, and generally reversible.

Thus, although clinical trials have investigated novel treatments for NET patients experiencing treatment failure and disease progression, data are limited on second and successive lines of treatment and are even sparser for high-grade pNET, which is usually excluded from clinical trials. The RADIANT-3 trial enrolled patients who had previously received antineoplastic therapy; 49 and $50 \%$ of the everolimus-treated population received 
previous somatostatin analogue therapy and chemotherapy, respectively [10]. Here, we describe prolonged clinical response to everolimus plus octreotide long-acting repeatable (LAR) as treatment in a patient with high-grade advanced pNET in the late-line therapy setting.

\section{Case Report}

In June 2008, a 34-year-old woman presented with a 2-month history of epigastric and periumbilical pain, fatigue, anorexia, nausea, and emesis. Physical assessment showed mild abdominal distention with painful hepatomegaly extending to the left hypochondrium, no adenopathy, and normal findings on echocardiogram (left ventricular ejection fraction, $72 \%$ ). Computed tomography (CT) revealed hepatomegaly with multiple lesions (the largest lesion measured approximately $11.5 \mathrm{~cm}$ in diameter), a $5 \times 7-\mathrm{cm}$ mass in the tail of the pancreas, and a collection of subcapsular hepatic, perihepatic, and perivascular fluid. An analysis of tumoral tissue obtained by liver biopsy showed electron-dense granules containing synaptophysin and chromogranin A (CgA), high mitotic count (30/10 high-power fields), and high expression of Ki-67 (found in 30-40\% of tumor cells) (fig. 1). Octreotide scintigraphy confirmed somatostatin receptor expression in the pancreatic tumor and in liver and peritoneal metastases. At the time of diagnosis, the patient's Karnofsky performance status (KPS) was 70\%.

Chemotherapy was initiated with streptozocin and 5-fluorouracil in July 2008; the patient received 3 cycles of this first-line chemotherapy. In September 2008, clinical and biomarker evaluation demonstrated progressive disease, and treatment was switched to second-line cisplatin and etoposide for 4 cycles. After disease progression, the patient began treatment with streptozocin and doxorubicin. After the completion of 3 cycles, however, the disease continued to progress rapidly, as assessed by radiologic imaging and changes in biomarker levels (CgA value, 4,381 ng/ml) (table 1).

After 9 months of chemotherapy and 3 lines of therapy, the patient had a KPS of 60\%, peritoneal metastases, and palpable hepatomegaly. She required high-dose opioids to achieve analgesia. Fourth-line treatment with temozolomide $\left(150 \mathrm{mg} / \mathrm{m}^{2}\right.$ on days $\left.9-14\right)$ and capecitabine $\left(1,000 \mathrm{mg} / \mathrm{m}^{2}\right.$ for 14 days every 3 weeks) was subsequently initiated and led to the achievement of partial radiologic response at 12 weeks ( 4 cycles); however, the patient experienced disease progression after 6 cycles of therapy.

In September 2009, after receiving 1 dose of fifth-line weekly paclitaxel, the patient was hospitalized because of toxicity and general deterioration in health. She had uncontrolled pain, esophagitis, and small bowel partial obstruction caused by peritoneal disease progression that prevented her from eating without severe nausea. $\operatorname{CgA}$ concentration increased to $4,600 \mathrm{ng} / \mathrm{ml}$, KPS decreased to $50 \%$, and CT demonstrated massive hepatic and peritoneal tumor infiltration (fig. 2a). Therefore, the patient was given intravenous fluids, morphine, scopolamine butyl bromide, dexamethasone, and subcutaneous octreotide as palliative and conservative therapy. After 1 week, she was able to tolerate oral fluids and drugs and experienced some improvement in general state. Taking into account initial data findings from the recent RADIANT-2 study [11], the patient's age, and her request to receive further lines of treatment, everolimus $10 \mathrm{mg}$ /day plus octreotide LAR $30 \mathrm{mg}$ every 4 weeks were initiated upon patient informed consent and compassionate use guidelines. Octreotide LAR had not previously been administered because of the patient's high baseline Ki-67 index $(30-40 \%)$. 
Fifteen days after the initiation of sixth-line therapy with everolimus plus octreotide LAR, the CgA concentration decreased to $1,388 \mathrm{ng} / \mathrm{ml}$. The patient achieved partial radiologic response ( $>50 \%$ decrease in tumor size) after 2 months of treatment (fig. $2 \mathrm{~b}$ ). By January 2010, her general health status had markedly improved, corresponding to a KPS of $90 \%$, with pain and oral restrictions alleviated. Clinically important partial response (decrease in tumor size $>80 \%$ ) was confirmed by CT (as per RECIST v1.0; fig. 2c) and by reduced CgA levels after 8 months of therapy with everolimus (fig. 3). The lowest CgA value, $137 \mathrm{ng} / \mathrm{ml}$, was achieved in August 2010 after approximately 1 year of treatment.

Throughout the course of everolimus plus octreotide LAR treatment, the patient experienced grade 1 hyperglycemia and grade 2 anemia, which were treated with an antidiabetic diet and erythropoietin plus intravenous iron therapy, respectively. Grade 1 stomatitis was also documented but did not require pharmacologic intervention. After 1 year of treatment, the patient reported sporadic grade 1 emesis, grade 1 diarrhea, and grade 1 hand-foot syndrome.

In December 2010, after 15 months of everolimus and octreotide LAR as sixth-line therapy, the patient continued to be asymptomatic and had a KPS of $90 \%$. Assessment by CT demonstrated an increase in the size of a peritoneal node (from $17 \mathrm{~mm}$ in early scans to 25 $\mathrm{mm})$. To prevent disease progression, everolimus was administered at a higher dose (15 $\mathrm{mg}$ /day), and 1 month later the node had decreased in size (to $21 \mathrm{~mm}$ ).

After more than 1 year of treatment benefit with everolimus, during CT evaluation in March 2011, the patient's liver metastases were shown to have progressed. A diagnostic biopsy was repeated. However, it was possible only to confirm the high Ki-67 index and electron-dense granules containing synaptophysin and CgA. Most of the cells were necrotic, and it was not possible to check for tissue expression of potential everolimus response predictors such as mTOR and 4EB1. One week later clinical progression was reported, and 2 weeks later the patient was hospitalized with liver failure, fever, jaundice, painful hepatomegaly, extrinsic compression of the stomach, anorexia, and asthenia. After progressive deterioration in her general health, the patient died on April 4, 2011, 33 months after the initial diagnosis and 19 months after the initiation of treatment with everolimus plus octreotide LAR.

\section{Discussion}

In the case presented here, a patient with high-grade pNET and 5 failed chemotherapy regimens was treated with everolimus plus octreotide LAR and achieved prolonged objective clinical and radiologic response, including improvement in hepatic and peritoneal metastatic sites, reduction in disease progressive biomarkers, and resolution of constitutional symptoms with improved KPS. The radiologic response persisted for more than 15 months, and CT assessment revealed the important partial radiologic response of $>80 \%$ decrease in tumor size after 4 months of initiation of everolimus plus octreotide LAR therapy. The regimen was well tolerated by the patient, and adverse events (hyperglycemia, sporadic emesis, diarrhea, and hand-foot syndrome) were generally manageable and grade 1 in severity.

The combination of everolimus plus octreotide LAR demonstrated markedly improved clinical benefit compared with previous first- to fifth-line treatment regimens administered to the patient (table 1). Partial radiologic response was achieved only at 12 weeks with fourth-line temozolomide and capecitabine, but rapid disease progression was observed in $<6$ months with this regimen and in $<3$ months with other therapy lines. One explanation for 
the efficacy of temozolomide and capecitabine is the decreased expression of the DNA repair enzyme 06-methylguanine-DNA methyltransferase (MGMT) in pNET. Future studies should investigate whether low MGMT expression in metastatic pNET is a predictive factor that correlates with response to capecitabine and temozolomide [12]. Treatment with everolimus plus octreotide LAR, on the other hand, resulted in dramatic clinical and CT imaging responses and PFS of 19 months.

The patient in this study derived a clinical benefit with everolimus-based therapy similar to the median values reported in the RADIANT trials, which enrolled patients with lowgrade NET [10]. In the phase III RADIANT-3 trial, patients with pNET receiving $10 \mathrm{mg}$ everolimus achieved a median PFS of 11 months, and $34 \%$ of patients were progression free at 18 months [10]. Treatment with the same dose of everolimus plus octreotide LAR in this case study prolonged PFS to approximately 15-19 months, which is similar to the results reported in RADIANT-2 [11]. Only $4.8 \%$ of patients experienced partial response and $64 \%$ minor tumor shrinkage (not meeting RECIST v1.0 criteria) in RADIANT-3 [10], whereas our patient achieved major tumor shrinkage with everolimus plus octreotide LAR. The clinical and radiologic benefits were surprising considering that a poorer clinical outcome might have been expected given the disease stage, high-grade tumor, 5 failed chemotherapies, and poor KPS.

Neither RADIANT-3 nor RADIANT-2 included objective tumor response as a secondary endpoint, whereas the PROMID study was a phase III trial investigating the antitumor effects of octreotide LAR in patients with metastatic neuroendocrine midgut tumors [13]. Median time to tumor progression was 14.3 months (95\% CI, 11.0-28.8 months) and 6.0 months (95\% CI, 3.7-9.4 months) in patients who received octreotide LAR and placebo, respectively [12]. The PROMID patient population was drug naive and excluded those with a prolonged ( $>4$ weeks) history of previous therapies. The PROMID and RADIANT-2 studies included patients with well-differentiated midgut tumors, which are indolent $[11,13]$. The estimated time to tumor progression for our patient was similar to that reported for patients in PROMID, but the tumor regression seen in our patient was better than that reported for those patients [12].

Our patient tolerated everolimus plus octreotide LAR as a sixth-line regimen for the management of high-grade pNET with minimal toxicity, similar to what has been reported in the RADIANT-3, RADIANT-2, and PROMID trials $[11,13]$. Treatment strategies associated with reduced toxicity, as demonstrated with everolimus-based therapy, become particularly important in the late-line setting because health-related quality of life often outweighs clinical benefit in patients with reduced lifespans.

The effect of everolimus in poorly differentiated tumors is unknown; these tumors are usually excluded from clinical trials because of their aggressiveness. However, these tumors have a better response rate to chemotherapy and may express more mTOR than welldifferentiated tumors, and mTOR elevation appears to be consistent across the primary tumor site $[14,15]$. Although there are no published studies that confirm it, everolimus may be a valuable treatment option in patients with poorly differentiated NET, either before the initiation of chemotherapy to sensitive tumor cells or after chemotherapy to help maintain response over time. It should be noted that the only chemotherapy regimen with confirmed activity in this setting is cisplatin and etoposide.

Treatment with everolimus plus octreotide LAR demonstrated definitive clinical response and prolonged PFS of more than 15 months in this heavily pretreated patient with high-grade pNET. Despite this regimen having been a sixth-line therapy, it improved clinical benefit with minimal toxicity compared with the 5 other previous lines of treatment the patient had received. In summary, this case study suggests the combination of everolimus 
Fonseca et al.: Prolonged Clinical Benefit of Everolimus Therapy in the Management of High-Grade Pancreatic Neuroendocrine Carcinoma

plus octreotide LAR may offer a valuable treatment strategy for patients with heavily pretreated, advanced, high-grade pNET.

\section{Acknowledgements}

We thank Inés Lorenzo Lozano and Carla Uriburu for assistance with translation. Financial support for medical editorial assistance was provided by Novartis Pharmaceuticals. We thank Clare Lee and Jennifer M. Kulak, PhD, for their medical editorial assistance with the manuscript.

\section{Disclosure Statement}

The authors declare that they have no conflicts of interest.

\section{References}

1 Yao JC, Eisner MP, Leary C, Dagohoy C, Phan A, Rashid A, Hassan M, Evans DB: Population -based study of islet cell carcinoma. Ann Surg Oncol 2007;14:3492-3500.

$\longrightarrow 2$ Oberg K, Akerstrom G, Rindi G, Jelic S: Neuroendocrine gastroenteropancreatic tumours: ESMO Clinical Practice Guidelines for diagnosis, treatment and follow-up. Ann Oncol 2010;21(suppl 5):v223-v227.

-3 Yao JC, Hassan M, Phan A, Dagohoy C, Leary C, Mares JE, Abdalla EK, Fleming JB, Vauthey JN, Rashid A, Evans DB: One hundred years after 'carcinoid': epidemiology of and prognostic factors for neuroendocrine tumors in 35,825 cases in the United States. J Clin Oncol 2008;26:3063-3072.

4 Kouvaraki MA, Ajani JA, Hoff P, Wolff R, Evans DB, Lozano R, Yao JC: Fluorouracil, doxorubicin, and streptozocin in the treatment of patients with locally advanced and metastatic pancreatic endocrine carcinomas. J Clin Oncol 2004;22:4762-4771.

-5 Delaunoit T, Ducreux M, Boige V, Dromain C, Sabourin JC, Duvillard P, Schlumberger M, de Baere T, Rougier P, Ruffie P, Elias D, Lasser P, Baudin E: The doxorubicin-streptozotocin combination for the treatment of advanced well-differentiated pancreatic endocrine carcinoma: a judicious option? Eur J Cancer 2004;40:515-520.

6 Kulke MH, Bendell J, Kvols L, Picus J, Pommier R, Yao J: Evolving diagnostic and treatment strategies for pancreatic neuroendocrine tumors. J Hematol Oncol 2011;4:29.

7 Eriksson B, Annibale B, Bajetta E, Mitry E, Pavel M, Platania M, Salazar R, Plöckinger U; on behalf of Mallorca Consensus Conference participants and the European Neuroendocrine Tumor Society: ENETS Consensus Guidelines for the Standards of Care in Neuroendocrine Tumors: chemotherapy in patients with neuroendocrine tumors. Neuroendocrinology 2009;90:214-219.

8 Strosberg JR, Coppola D, Klimstra DS, Phan AT, Kulke MH, Wiseman GA, Kvols LK; for the North American Neuroendocrine Tumor Society: The NANETS consensus guidelines for the diagnosis and management of poorly differentiated (high-grade) extrapulmonary neuroendocrine carcinomas. Pancreas 2010;39:799800.

-9 Yao JC, Phan AT, Chang DZ, Wolff RA, Hess K, Gupta S, Jacobs C, Mares JE, Landgraf AN, Rashid A, MericBernstam F: Efficacy of RAD001 (everolimus) and octreotide LAR in advanced low- to intermediate-grade neuroendocrine tumors: results of a phase II study. J Clin Oncol 2008;26:4311-4318.

10 Yao JC, Shah MH, Ito T, Bohas CL, Wolin EM, Van Cutsem E, Hobday TJ, Okusaka T, Capdevila J, de Vries EG, Tomassetti P, Pavel ME, Hoosen S, Haas T, Lincy J, Lebwohl D, Öberg K; for the RAD001 in Advanced Neuroendocrine Tumors, Third Trial (RADIANT-3) Study Group: Everolimus for advanced pancreatic neuroendocrine tumors. N Engl J Med 2011;364:514-523.

11 Pavel M, Hainsworth JD, Baudin E, Peeters M, Hörsch D, Winkler RE, Klimovsky J, Lebwohl D, Jehl V, Wolin EM, Oberg K, Van Cutsem E, Yao JC; for the RADIANT-2 Study Group: Everolimus plus octreotide long-acting repeatable for the treatment of advanced neuroendocrine tumors associated with carcinoid syndrome (RADIANT-2): a randomised, placebo-controlled, phase 3 study. Lancet 2011;378:2005-2012.

12 Strosberg JR, Fine RL, Choi J, Nasir A, Coppola D, Chen DT, Helm J, Kvols L: First-line chemotherapy with capecitabine and temozolomide in patients with metastatic pancreatic endocrine carcinomas. Cancer 2011;117:268-275.

13 Rinke A, Muller HH, Schade-Brittinger C, Klose KJ, Barth P, Wied M, Mayer C, Aminossadati B, Pape UR, Bläker M, Harder J, Arnold C, Gress T, Arnold R; for the PROMID Study Group: Placebo-controlled, double- 


\section{Case Reports in Oncology}

\begin{tabular}{l|l}
\hline Case Rep Oncol 2013;6:441-449 \\
\hline DOI: 10.1159/000354754 & $\begin{array}{l}\text { ○ 2013 S. Karger AG, Basel } \\
\text { www.karger.com/cro }\end{array}$ \\
\hline
\end{tabular}

Fonseca et al.: Prolonged Clinical Benefit of Everolimus Therapy in the Management of High-Grade Pancreatic Neuroendocrine Carcinoma

blind, prospective, randomized study on the effect of octreotide LAR in the control of tumor growth in patients with metastatic neuroendocrine midgut tumors: a report from the PROMID Study Group. J Clin Oncol 2009;27:4656-4663.

14 Shida T, Kishimoto T, Furuya M, Nikaido T, Koda K, Takano S, Kimura F, Shimizu H, Yoshidome H, Ohtsuka M, Tanizawa T, Nakatani Y, Miyazaki M: Expression of an activated mammalian target of rapamycin (mTOR) in gastroenteropancreatic neuroendocrine tumors. Cancer Chemother Pharmacol 2010;65:889-893.

15 Catena L, Bajetta E, Milione M: Mammalian target of rapamycin expression in poorly differentiated endocrine carcinoma: clinical and therapeutic future challenges. Targeted Oncol 2011;6:65-68.

Table 1. Lines of treatment administered from June 2008 to March 2011

\begin{tabular}{llll}
\hline Line & Treatment & $\begin{array}{l}\text { Time to progression, } \\
\text { months }\end{array}$ & Best response \\
\hline First & Streptozocin + 5-fluorouracil & 2.5 & Stable disease \\
Second & Cisplatin + etoposide & 4 & Minimum response \\
Third & Streptozocin + doxorubicin & 2.5 & Progressive disease \\
Fourth & Temozolomide + capecitabine & 5 & Partial response \\
Fifth & Paclitaxel & 0 & - \\
Sixth & Everolimus + octreotide LAR & 19 & Partial response \\
\hline
\end{tabular}
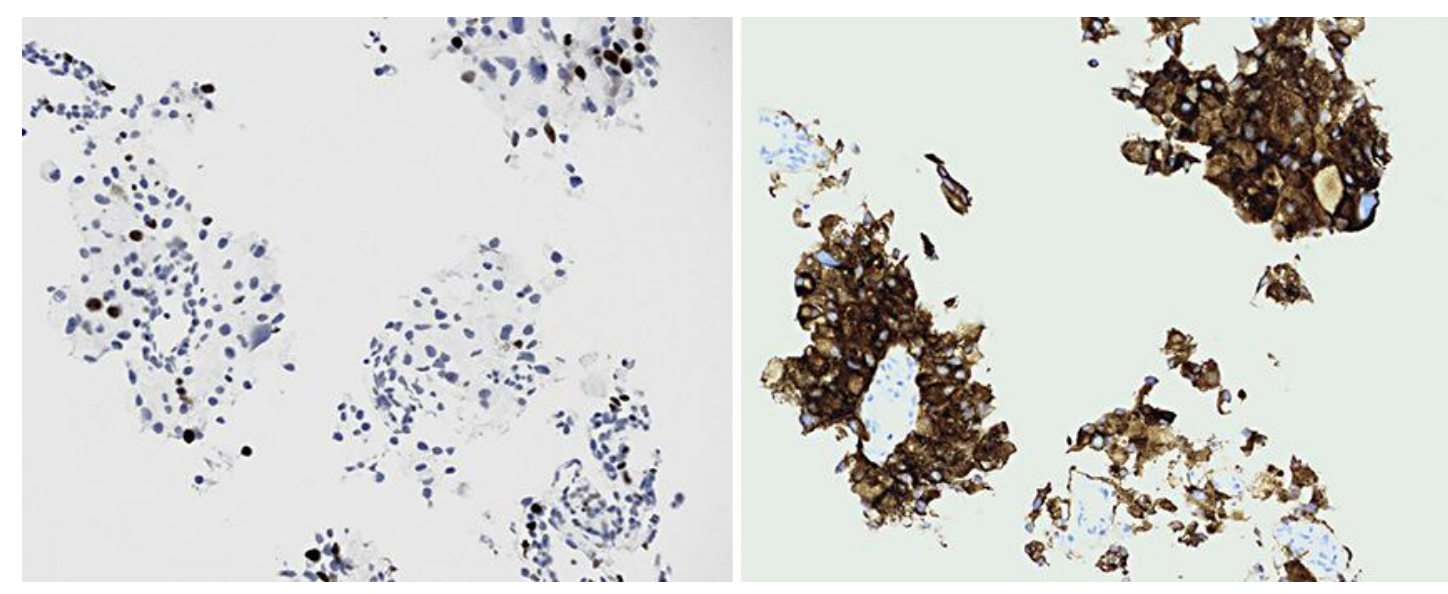

Fig. 1. Ki-67 (left) and synaptophysin (right) staining of pNET liver biopsy. 


\section{Case Reports in Oncology}

\begin{tabular}{l|l}
\hline Case Rep Oncol 2013;6:441-449 & \\
\hline DOI: 10.1159/000354754 & $\begin{array}{l}\text { ○ 2013 S. Karger AG, Basel } \\
\text { www.karger.com/cro }\end{array}$ \\
\hline
\end{tabular}

Fonseca et al.: Prolonged Clinical Benefit of Everolimus Therapy in the Management of High-Grade Pancreatic Neuroendocrine Carcinoma
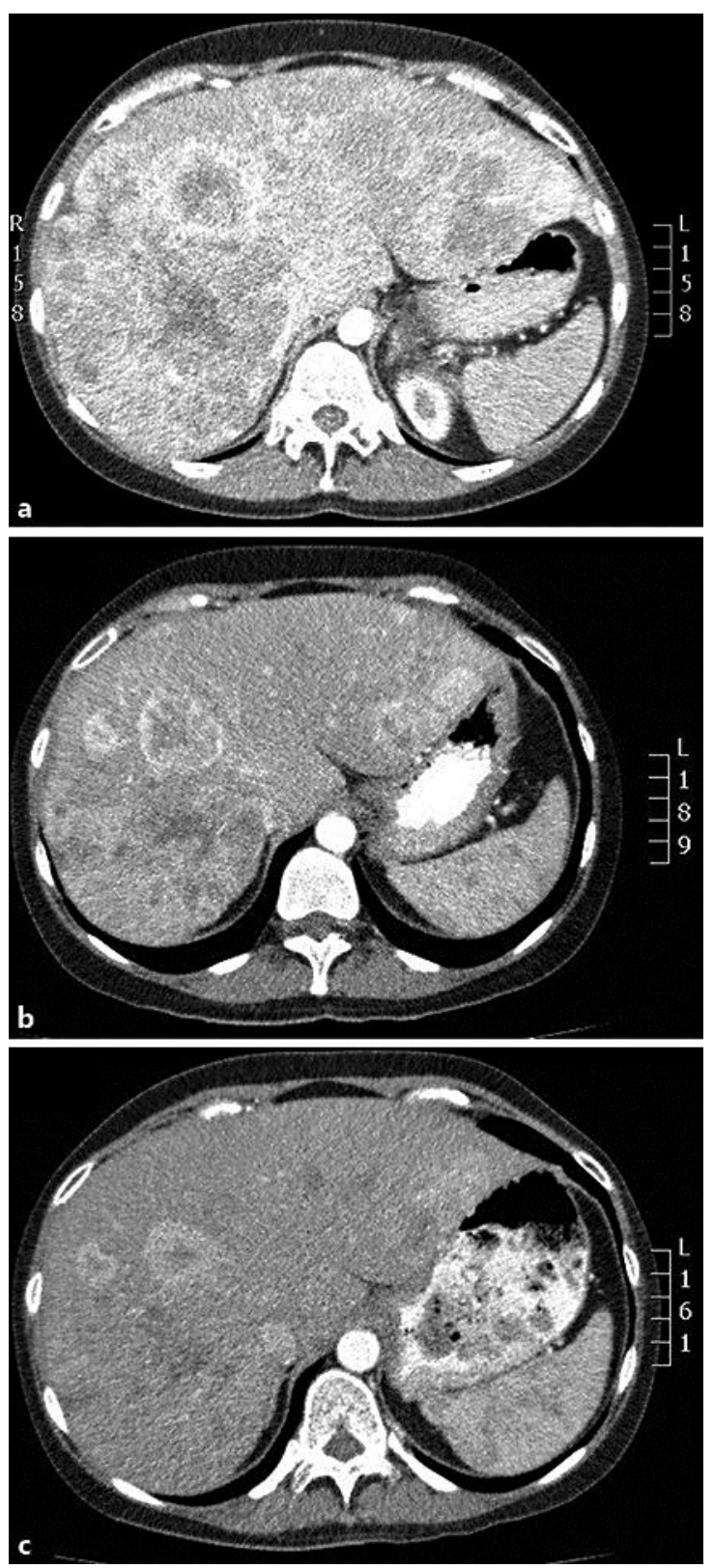

Fig. 2. CT scans of the abdomen. a September 2009, after fifth-line paclitaxel. b November 2009, 2 months after the initiation of everolimus and octreotide LAR. c January 2010, partial response confirmed $(>80 \%$ reduction in size per RECIST v1.0 criteria). 


\section{Case Reports in Oncology}

Fonseca et al.: Prolonged Clinical Benefit of Everolimus Therapy in the Management of High-Grade Pancreatic Neuroendocrine Carcinoma

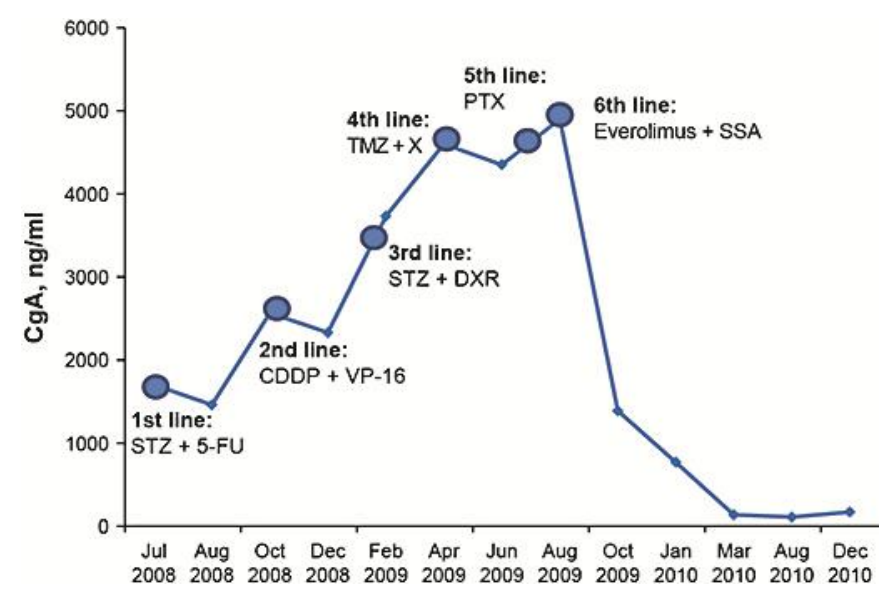

Fig. 3. CgA variation over 6 lines of therapy. CDDP = Cisplatin; DXR = doxorubicin; 5-FU = 5-fluorouracil; PTX = paclitaxel; SSA = somatostatin analogue; STZ = streptozocin; TMZ = temozolomide; VP-16 = etoposide; $\mathrm{X}=$ capecitabine. 